\title{
An Effective Automated Technique for Retinal Disease Identification in Diabetic Retinopathy without Manually Labeled Kit
}

\author{
Romana Naznin \\ B.Tech in Electronics and Communication \\ Dept. of ECE EIILM University \\ Sikkim, India
}

\author{
Ipsita Parida \\ B.Tech in Electronics and Communication \\ Dept. of ECE EIILM University \\ Sikkim, India
}

\begin{abstract}
Diabetes is a rapidly increasing common disease among people in worldwide which causes dysfunction of various organs. Diabetic retinopathy (DR) is the main cause of blindness in adults. Exudates are among the primary symptoms of diabetic retinopathy. So, regular screening is needed for early detection of exudates which could decrease the chances of blindness. The quick growth of diabetes pushes the limits of the current DR screening capabilities for which the digital imaging of the eye fundus algorithm automatic image analysis algorithm is the conventional solution. In this work, new exudates detection method is given which has overcome the limitations of labeled lesion training sets such as: time consumption, complexity and more probability of error. In this we present a new concept to normalize the fundus image and directly compare our method with an implementation. Here, we introduce two variations of a new exudates segmentation method that comes under the category of thresholding methods and find out the diabetics as well as other eye diseases.
\end{abstract}

\section{Keywords}

DR, Exudates segmentation method, Ophthalmology, Diabetic macular edema (DME), Image normalization, Preprocessing

\section{INTRODUCTION}

Ophthalmology is an important branch of biomedical field which needs computer-aided automated techniques for pathology identification in human eye. The focus of this work is on the procedure of automated technique used in the identification of retinal sickness in diabetic retinopathy. DR is a common visional sickness and main cause of vision loss. Diabetic macular edema (DME) is a problem of DR. DME arises from swelling of the retina in diabetic patients dripping of fluid from blood vessels within the macula. Exudates and fat leakages appear as noticeable indication of retinal defect in DR. However, manual checkup by ophthalmologist takes more time and the number of experts is not satisfactory to meet the requirement of screening. Currently, imaging the eye fundus with modern techniques is a common practice in man eye clinics. Eye fundus imaging is considered a non-invasive and painless method to screen. Digital imaging of the eye fundus and automatic or semi-automatic image analysis algorithm based on image processing and compute vision techniques offer a great prospective.

The methods for exudate segmentation can be approximately distributed into four different categories. Thresholding methods base the exudate reorganization on an overall or adaptive grey level analysis [2,3]. Region growing methods segment the images using the special contiguity of gray levels [4].Morphology methods employ grey scale morphological operators to identify all structures with estimated shape. These structures are removed from the image so the exudates can be identified [5, 6]. Classification methods build a feature vector for each pixel or pixel cluster.

\section{OVERVIEW OF THE PRESENT WORK}

In this work two methods presented are divided in two parts: Preprocessing (illumination correction and contrast enhancement) and Exudate detection (Segmentation of different features and Detection of lesions).Below figure (1) shows an example of a fundus image, the intermediate and final steps of these techniques.

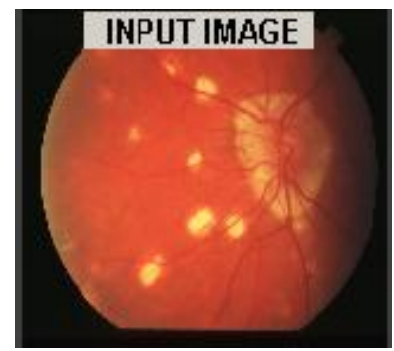

Original Image

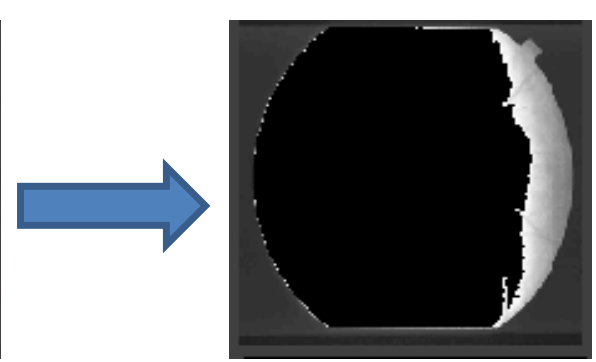

Extracting Green Channel

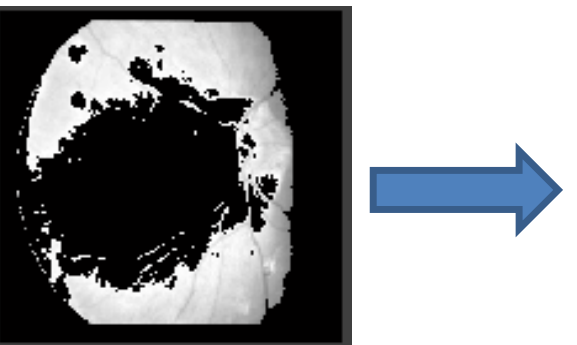

Image Normalization 


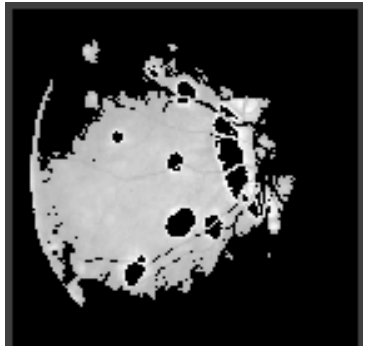

Edge Detected Image
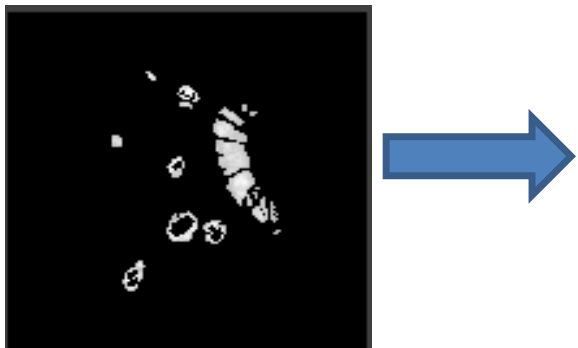

Exudates segmented retinal Image.

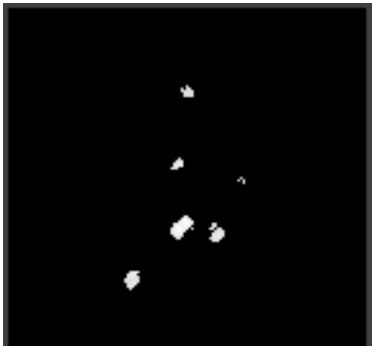

Exudate Probability

Figure: (1) Automatic Segmentation of Exudates Detection

\subsection{Algorithm for Segmentation}

Input: Pixel $\left\{\mathrm{x}_{\mathrm{i}}, \mathrm{y}_{\mathrm{i}}\right\}_{\mathrm{i}=1}^{\mathrm{M}}$, where $\mathrm{x}_{\mathrm{i}}=\left(\mathrm{x}_{\mathrm{g}}, \mathrm{x}_{1}, \mathrm{x}_{\mathrm{y}}\right), \mathrm{y}_{\mathrm{i}} €\{-1,1\}$.Pixels' weights

$\mathrm{D}_{1}(i)=1 / \mathrm{M}$

1: Update all $T$ weak learners in the feature pool with the input data

2: Initialize $\mathrm{w}_{0}=0$

3: for $\mathrm{t}=1$ to $T$ do

4: $\quad \mathrm{h}_{\mathrm{t}}=\arg \min \varepsilon_{\mathrm{K}}=\sum_{\mathrm{i}=1}^{\mathrm{M}} \mathrm{D}_{\mathrm{t}}(i)\left[\mathrm{y}_{\mathrm{i}} \neq \mathrm{h}_{\mathrm{k}}\left(\mathrm{x}_{\mathrm{i}}\right)\right]$

$$
\mathrm{h}_{\mathrm{k}} € \mathrm{~h}
$$

5: $\quad$ Compute $\mathrm{u}_{\mathrm{t}}$ and $\mathrm{e}_{\mathrm{t}, \mathrm{i}}$, where $i=\arg \min \varepsilon_{\mathrm{k}}$

6: $\quad$ Set $\alpha_{\mathrm{t}}=1 / 2 \log 1-\varepsilon_{\mathrm{t}}-$

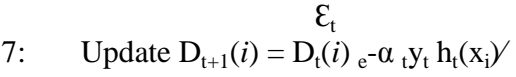

8: $\quad \mathrm{W}_{\mathrm{t}} \leftarrow \mathrm{W}_{\mathrm{t}^{-1}}+\alpha_{\mathrm{t}} \mathrm{u}_{\mathrm{t}} \mathrm{e}_{\mathrm{t},(i)}$

9: end for

Output: $w_{B}=w_{T}$ for soft segmentation

\subsection{Preprocessing}

\subsubsection{Background Estimation}

This approach uses green channel I9 of the image and the Ii channel from the HSI color space. The initialization of the analysis takes place by manipulating the background with normalization has taken place with morphological reform as opposite to the more common practice of subtracting the median filtered re-sult, which appears to develop the elimination of nerve fiber layer and other structures at the edges of the optic nerve $(\mathrm{ON})$. The pseudo code of algorithm shows this step. Huge median filter whose size is 3:1:0 the height to get a normalized version. In this method,

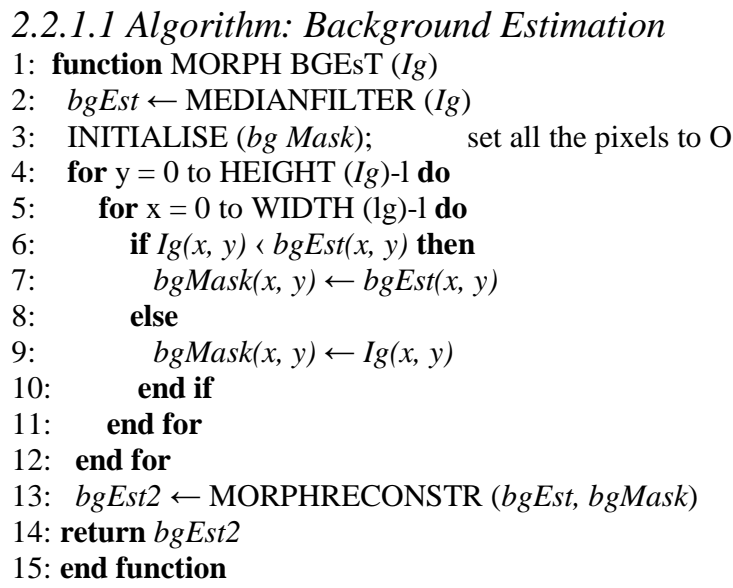

\subsubsection{Image Normalization}

Once the background bgEst is evaluated, it is deducted from the original image with signed precision. In the resulting image, the highest peak of the histogram is always centered on zero regardless of the ethnicity of the patient or disease strat-ification. The histogram indicates a clear difference between dark structures and bright structures. The former signifies the vasculature, macula, dark lesions and other structures and their distribution varies depending on the ethnicity or pigmentation of the patient. Bright structures are originated in the positive side of the histogram and include the ON, bright lesions, and other structures. The distribution is fairly constant across various ethnicities. Because of the features of the normalized image, all the exudate candidates can be selected Icand with a hard threshold thcand. This has clear computational benefits in comparison with model fitting processes which are more delicate to suboptimal background deduction and do not suggest clear inspirations for threshold selection. In this case thcand $=3$ is used simply by empirically taking a value little above 0 in order to provide less background estimation errors.

The pre-processing step of $\mathrm{ON}$ elimination looks to be a constant across all the methods in the literature. This is clearly given the potential color resemblance between $\mathrm{ON}$ and exudates in fundus Since the ON detection is a advanced method and our motive to compare the exudate detection only, an automatic ON the improvement of the images detection was not implemented for this experiments. Instead, the ON was eliminated manually and covered out a region a little greater than the average ON size [1]

\subsection{Exudate Detection}

The exudate detection is done by allocating a score for each exudate candidate. The exudate candidates are nominated by running a 8-neighbour connected component analysis on Icand. Here, the implementation has taken place by two ways to allocate this score, one based on Kirsch's Edges [7] and the other based on Stationary Wavelets. Both approaches pursue to take benefit of the greater inner and outer edge values of exudates in comparison to non-exudate structures

\subsubsection{Kirsch's Edges}

Kirsch edge exposure is used to eliminate the blood vessels by using threshold values. The Kirsch operator is a non-linear edge indicator that achieves the maximum edge strength in a few preset instructions. The kirsch edge detector not only describes the presence of edges but also it defines the direction in which the edge transfers. The masks of this Kirsch technique are defined by taking a single mask and revolving it to eight main compass directions such as North, Northwest, West, Southwest, South, Southeast, East and Northeast, shows in fig.(b). The 
average edge outputs of I kirsch under each lesion cluster are manipulated and given to the lesion in its entirety. The thresholds used to estimate the final output are thfin $\mathrm{E}$ \{O: 0.5: $30\}[1]$.

\subsubsection{Stationary Wavelets}

A stationary Haar wavelet study is performed up to the second level on Ig.The process is inverted maintaining the last vertical, diagonal and horizontal details only, as these are the wavelet co efficient that seem to contain most of the foreground structures.

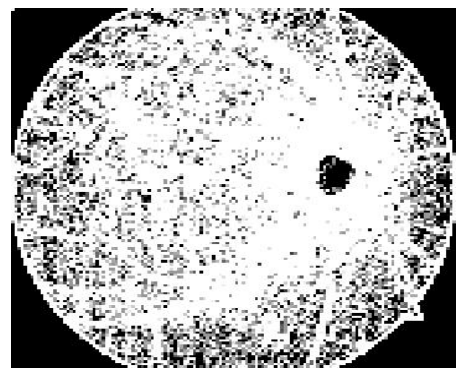

Fig.(a). Edge Detected Image

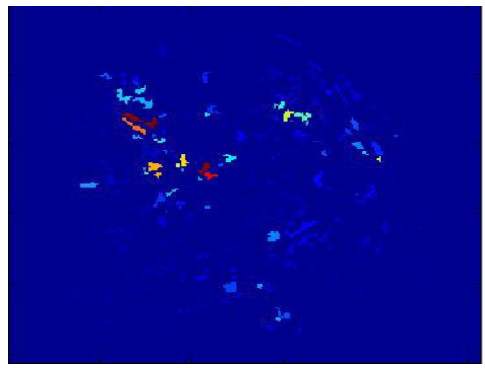

Fig.(b).Exudates segmented retinal image.

The Kirsch edge detection algorithm uses a $3 \times 3$ table of pixels to store a pixel and its neighbors while calculating the derivatives. The $3 \times 3$ table of pixels is called a convolution table, because it moves across the image in a convolution-style algorithm. Kirsch edges try to capture the external edges of the lesion candidate. The average edge outputs under each lesion cluster are calculated and assigned to the lesion in its entirety. Finally, this algorithm provides segmented image which can clearly distinguish the exudates portion from the non-exudates pixels. The resultant image shown in fig b was exposed in color which clearly indicates the exudates pixels in the segmented retinal image[7].

This edge detector is based on the kernel k (shown below) evaluated at 8 different directions on Ig. The kernel outputs are combined together by selecting the maximum value found on each pixel output. The result is stored in the final I kirsch image.

$\mathrm{K}=\left|\begin{array}{ccc}\frac{5}{15} & -\frac{3}{15} & -\frac{3}{15} \\ \frac{5}{15} & 0 & -\frac{3}{15} \\ \frac{5}{15} & -\frac{3}{15} & -\frac{3}{15}\end{array}\right|$

By altering back to the image space we obtain a backgroundless image Iwav with a strong response at the center of exudates. Similarly to the former approach, we estimate the candidate by identifying variation in the Iwav area which corresponds to each lesion cluster of Icand as follows.

$$
\text { WaveScore }=\frac{\max \left(p x_{\text {wav }}\right)-\min \left(p x_{\text {wav }}\right)}{\max _{\text {wav }}}
$$

Where $\mathrm{px}_{\mathrm{wav}}$ are the pixels of $\mathrm{I}_{\mathrm{wav}}$ corresponding to a lesion candidate cluster. The thresholds used to estimate the final output are thfin

$$
\varepsilon=0: 0.05: I
$$

Retinal structures and HEs (Hard Exudates) seem more contrasted in the green channel component than those in the other channels of the color image. Hence, here the green channel Component is used in the fovea localization and HEs exposure was smoothed using two filtering stages; first by a median filter with a window size of $3 \times 3$ and then by a Gaussian filter with $=2$. The shade rectification was carried out using morphological processes. The background intensity is evaluated by applying morphological alternating opening and closing with the appropriate structuring element to be large enough to avoid entirely fitting within small candidate regions. The shade rectified image was produced by deducting this evaluated background from the smoothed image. A procedure of contrast enhancement is vital to improve the contrast of image features and HEs from the background. For this, a Contrast-Limited Adaptive Histogram Equalization (CLAHE) was applied to the result of the shade rectification.

CLAHE improves the image by altering the intensity values of the image. It operates on small regions instead of the whole image. The contrast of each small region is improved with histogram equalization. After the equalizations, the neighboring small regions are joined using bilinear interpolation.

Exposure of Hard Exudates In retinal images HEs commonly seem as bright regions with different boundaries. Two processes were carried out to detect HEs: adaptive thresholding and classification. The adaptive thresholding includes two steps: image partitioning into homogeneous regions and then segmenting candidates of HEs from the background of these regions. A classification method was carried out to classify HEs from non-HEs using a rule-based classifier.

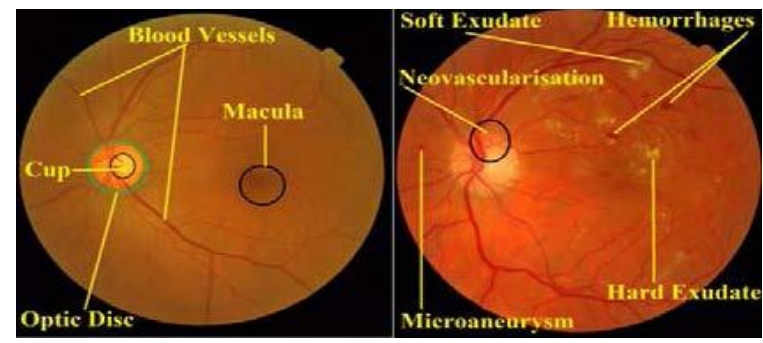

Figure. (c) Normal Retina and Figure. (d)Disease Retina[8]

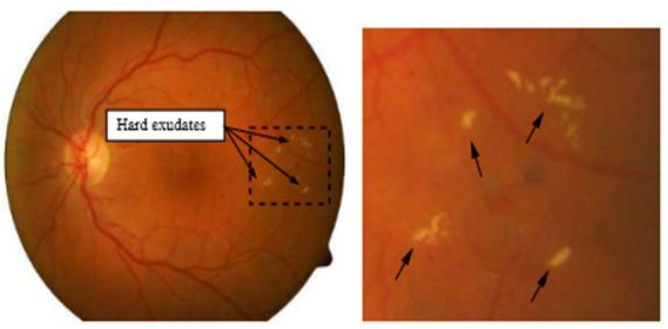

Figure. (e) Hard Exudate and Figure. (f)Close up image of the hard exudates $[9,10]$ 


\section{RESULT}

From the above experiment we note that the main aim of automated screening is identification procedure of the patient condition and segmentation is a step towards this objective. The mean square value (MSE) and peak signal to noise ratio (PSNR) of input digital image is calculated as presented in the below snapshot of the GUI model. Based on the value of MSE we can detect whether the person is suffering from diabetics or not and the diagnosis of other eye diseases can be found out from the PSNR value as shown in below table.

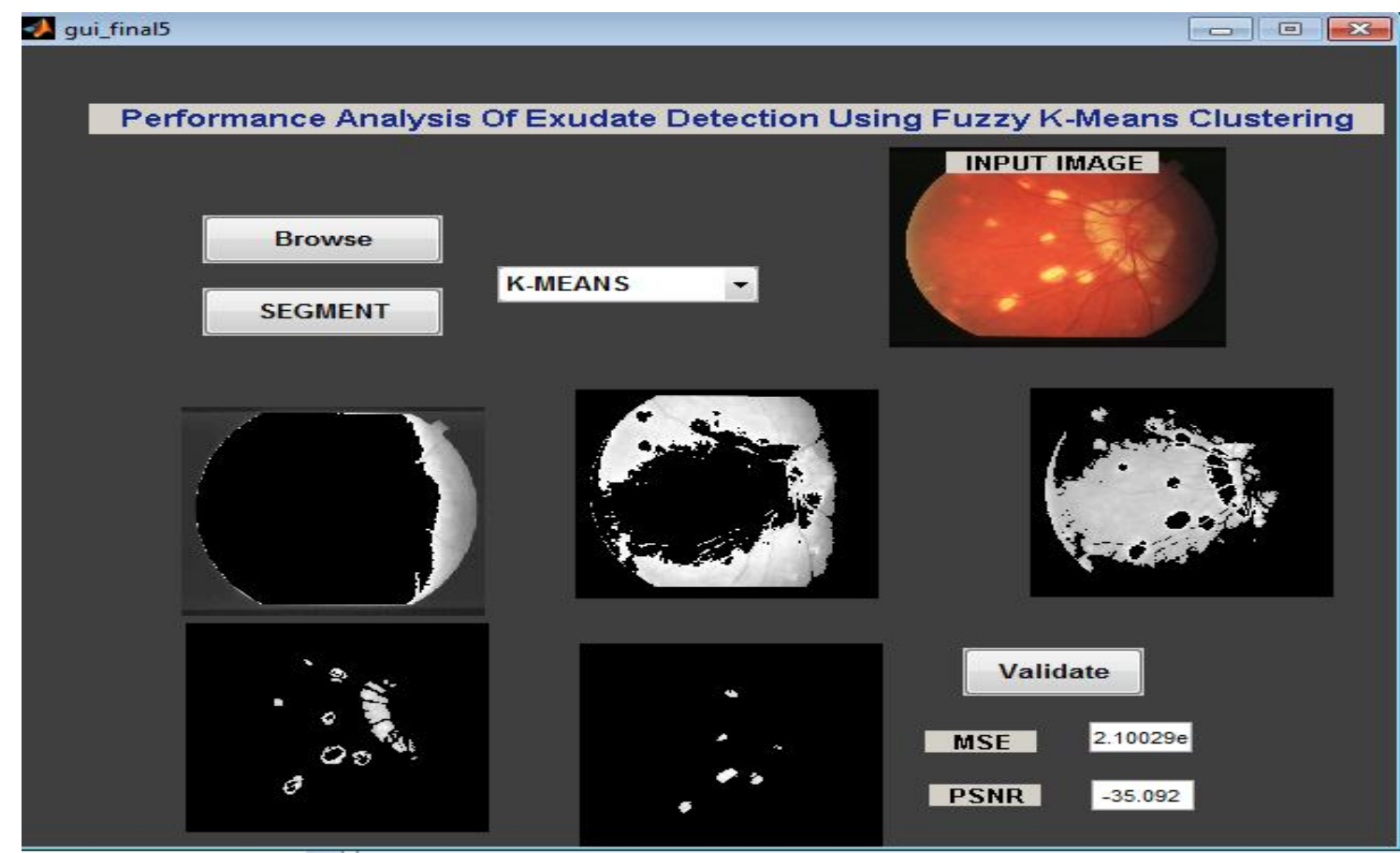

Table: 1 Observation output by using different samples (input image)

\begin{tabular}{|c|c|c|c|c|c|c|}
\hline \multirow[b]{2}{*}{$\begin{array}{l}\text { INPUT } \\
\text { IMAGE }\end{array}$} & \multicolumn{2}{|c|}{ MSE } & \multicolumn{2}{|c|}{ PSNR } & \multicolumn{2}{|c|}{ RESULT } \\
\hline & $\begin{array}{c}\text { FOR } \\
\text { NORMAL EYE }\end{array}$ & $\begin{array}{c}\text { FOR } \\
\text { ABNORMA } \\
\text { LEYE }\end{array}$ & $\begin{array}{c}\text { FOR } \\
\text { NORMAL } \\
\text { EYE }\end{array}$ & $\begin{array}{c}\text { FOR } \\
\text { ABNORMAL } \\
\text { EYE }\end{array}$ & $\begin{array}{l}\text { PRESENCE } \\
\text { OF } \\
\text { DIABETIC }\end{array}$ & $\begin{array}{c}\text { OTHERS } \\
\text { EYE } \\
\text { DISEASE }\end{array}$ \\
\hline & -10 to +10 & $2.10029 \mathrm{e}$ & -10 to +10 & -35.092 & NO & MYOPIA \\
\hline & -10 to +10 & $9.75235 \mathrm{e}$ & -10 to +10 & -31.7606 & NO & MYOPIA \\
\hline & -10 to +10 & $1.36127 \mathrm{e}$ & -10 to +10 & 36.201 & YES & $\begin{array}{c}\text { HYPER- } \\
\text { METROPI } \\
\text { A }\end{array}$ \\
\hline
\end{tabular}

The computational performances are estimated on Dual core $2.13 \mathrm{GHz}$ machine with 4GB of RAM. All the algorithms are implemented in Matlab code. This proposed methods need 2.3 and 1.7 seconds per image for the Wavelet and Kirsch's edges methods. 


\section{CONCLUSION}

In this work, we have implemented the novel automatic exudates segmentation method to detect the exudates from the retinal image of a diabetic patient at the earlier stage of diabetic retinopathy. Image segmentation systems clearly transform the appearance of eye fundus images to a more similar representation for eye disorders diabetic which is related to eye. This work is somewhat similar to the existing systems but additionally by calculated the PSNR and MNS from the retina image fundus and segment eye diseases also diagnosed. However, the image normalization procedure provides a significant computational advantage to our technique. When there are lots of image that ophthalmologists have to analysis, this method will give more suitable and faster approach with user-friendly interface, which they can input the eye image and the application will provide the output. The web based application would help ophthalmologists in order to detect the disease faster which implies to save patient from blindness. This application will very useful tool for the hospital in rural areas. Moreover, this application saves time and needs very low cost because we do not need much resource.

This list do not exactly covers all the work but absolutely it will make reader to understand the procedures and technique hired for diagnosis of exudates and this will help those who aspire to research.

\section{FEATURE SCOPE}

In long term, the system is better to have some progress on other platform such as mobile application. So that it would be easier to get access by people who use smartphone around the world.

\section{REFERENCE}

[1] L. Giancardo, F. Meriaudeau,T.P. Karnowski, Y. Li, K. W. Tobin Jr. , E. Chaum, MD, "Automatic Retina Exudates Segmentation Without a Manually Labelled training Set," International Journal of Advanced Research in Computer Science and Software Engineering,vol.3,Issue.3,March 2013.

[2] R Phillips, J Forrester, and P Sharp, "Automated detection and quantification of retinal exudates," Graefes
Arch Clin Exp Ophthalmology, vol. 231, no. 2, pp. 9094, Feb 1993.

[3] C I Sanchez, M Garcia, A Mayo, M I Lopez, and R Homero, "Retinal image analysis based on mixture models to detect hard exudates.," Medical Image Analysis, vol. 13, no. 4, pp. 650-658, Aug 2009.

[4] C Sinthanayothin, J F Boyce, T H Williamson, H L Cook, E Mensah, S Lal, and D Usher, "Automated detection of diabetic retinopathy on digital fundus images," Diabetic Medicine, vol. 19, no. 2, pp. 105-112, Feb 2002.

[5] T Walter, J K Klein, P Massin, and A Erginay, "A contribution of image processing to the diagnosis of diabetic retinopathy detection of exudates in color fundus images of the human retina.," IEEE Transactions on Medical Imaging, vol. 21, no.10,pp. 1236-1243, Oct 2002.

[6] Akara Sopharak, Bunyarit Uyyanonvara, Sarah Barman, and Thomas $\mathrm{H}$ Williamson, "Automatic detection of diabetic retinopathy exudates from non-dilated retinal images using Mathematical morphology methods." Computerized Medical Imaging and Graphics, vol. 32, no. 8, pp. 720-727, Dec 2008.

[7] National Eye Institute, National Institutes of Health. Available online at: http://www.nei.nih.gov/. [Referred 19.1.2010].

[8] Kade Mahesh k,"A Survey of Automated Techniques For Retinal Disease Identification in Diabetic Retinopathy.," International Journal of Advancements in Research \& Technology, Volume 2, Issue5, May-2013.

[9] Diabetic Retinopathy (online). Current Care Guideline. Working group set up by the Finnish Medical Society Duodecim and the Finnish Respiratory Society. Helsinki: Finnish Medical Society Duodecim, 2006 [referred 19.1.2010]. Available online at: www.kaypahoito.fi.com

[10] National Eye Institute, National Institutes of Health. Available online at: http://www.nei.nih.gov/. [Referred 19.1.2010]. 